\title{
El papel de las Ciencias Sociales frente a la intención de una reforma neoliberal del Estado paraguayo
}

\section{The role of the Social Sciences against the intention of a neoliberal reform of the Paraguayan State}

\author{
Luis Alexander Ovando ${ }^{a}$
}

\begin{abstract}
Resumen
La post-pandemia traerá consigo una serie de cambios en lo económico, político y social. Queda claro que habrá un antes y un después luego de la crisis sanitaria y que muchas de las modificaciones que vendrán, se darán en el campo de la administración pública. En lo que respecta a Paraguay, no es la excepción, el gobierno puso en marcha la reforma "estructural" del Estado, y en ella se aprecia una fuerte agenda neoliberal que como de costumbre, busca acompañar intereses empresariales por sobre otros. En este sentido y atendiendo que la emergencia sanitaria producirá considerables daños al tejido comunitario, el papel de las Ciencias Sociales en este contexto será de vital importancia, por un lado, para guiar las próximas medidas políticas hacia un enfoque más humano y por otro lado, para pensar en nuevos y mejores escenarios sociales posterior al COVID-19.
\end{abstract}

Palabras clave: reforma, neoliberalismo, ciencias sociales, pandemia.

\begin{abstract}
The post-pandemic will bring with it a series of economic, political and social changes. It is clear that there will be a before and after after the health crisis and that many of the changes that will come will take place in the field of public administration. With regard to Paraguay, it is not the exception, the government launched the "structural" reform of the State, and it shows a strong neoliberal agenda that, as usual, seeks to accompany business interests above others. In this sense and taking into account that the health emergency will cause considerable damage to the community fabric, the role of Social Sciences in this context will be of vital importance, on the one hand, to guide the next political measures towards a more humane approach and on the other hand, to think about new and better social scenarios after COVID-19.
\end{abstract}

a Universidad Nacional de Asunción, Facultad de Ciencias Sociales, Paraguay.

Correspondencia a: alexanderovando182@gmail. com

Recibido:

22 de mayo de 2020

Aceptado:

29 de junio de 2020

Artículo publicado en acceso abierto bajo la Licencia Creative Commons.

\section{c) (i)}

Cita:

Ovando, L. A., \& Riquelme, Q. (2020). El papel de las Ciencias Sociales frente a la intención de una reforma neoliberal del Estado paraguayo. Kera Yvoty: reflexiones sobre la cuestión social, 5 (número especial), 93-99.

Keywords: reform, neoliberalism, social sciences, pandemic. 


\section{Introducción}

El conocimiento científico, hoy por hoy, más que nunca, es fuente de información indispensable en la lucha contra el COVID-19. Tal vez, la medicina sea la ciencia más exigida en este momento, así también la economía y otras tantas áreas del saber. En este sentido, las ciencias sociales tampoco se quedan atrás. El entorno social, las desigualdades y la cultura, repercuten en el nivel de daño que se pueda obtener de la crisis sanitaria. Manejar estos elementos permite, por un lado, analizar los efectos comunitarios inmediatos y, por otro lado, sirven para pensar en nuevas formas de organización posterior a la pandemia.

Queda claro que la post pandemia traerá consigo a nivel mundial, una serie de reformas, tanto en lo político y económico, es en ese contexto donde las investigaciones sociales deben servir de guía para elaborar de la mejor forma posible, las políticas públicas encaminadas a mitigar las desigualdades que puedan derivar del coronavirus. Identificar los factores de riesgo, para así intervenir en ellas será el papel fundamental de las ciencias sociales, principalmente en los periodos de reestructuración o de reajustes que se darán posteriormente.

En lo que respecta a Paraguay, los cambios no tardaron en anunciarse. El gobierno puso en marcha la reforma "estructural" del Estado paraguayo, reforma que viene de la mano de un grupo político que por lo general no suele prestar mucha atención a las evidencias empíricas al momento de tomar decisiones importantes y que hasta el momento ha propuesto un conjunto de ajustes desde un punto de vista visiblemente neoliberal.

En resumidas palabras, los cambios a lo que hacen alusión las autoridades de turno se centran en descongestionar el aparato estatal a partir de la reducción de funcionarios públicos, recortes de sueldos, eliminación de ministerios y secretarias, además de la privatización de servicios estatales. Al mismo tiempo, este proyecto omite la idea de implementar nuevos impuestos en campos que podrían ayudar a contener los efectos futuros de la pandemia, para ser más precisos, en rubros como la soja, el tabaco el alcohol. Igualmente, la idea de una reforma agraria va quedando relegada a segundo plano, así como otros tantos temas de fuerte contenido social van siendo ignorados.

\section{Consideraciones para entender el carácter de la presente reforma}

Se suele afirmar que comunicamos con lo que decimos y también con lo que dejamos de decir, en este sentido, el hecho de evitar tratar nuevos paquetes tributarios, beneficiando con esto a algunos sectores industriales al no aumentarles los impuestos, no representa de ninguna forma un caso aisladoyajeno, sino que son parte de un proyecto ideológico que históricamente ha recelado en poner en juego los intereses empresariales de actores relacionados al poder político.

La intención de modificar el aparato estatal fue gestándose a finales del marzo pasado desde la iniciativa de figuras políticas relacionadas a la derecha conservadora. Al principio de abril, el poder ejecutivo mediante un decreto declaraba el Estado de emergencia sanitaria en todo el país y en ella autorizaba al Ministerio de Hacienda realizar un préstamo USS 1.600 millones que se destinarían para Salud, específicamente para inversión en insumos hospitalarios, para la duplicación de camas de terapias intensivas y adquisición de más reactivos para aumentar los test que detectan el COVID-19. Se contemplaba además un programa de contingencia para dar asistencia a unos 1.500.ooo trabajadores informales $y$ respaldo a las micros y medianas empresas.

Desde el gobierno sostenían que, el préstamo hecho no bastaría para contener los efectos provocados por la crisis sanitaria, así fue que se decretó el recorte del $10 \%$ del sueldo a funcionarios públicos que ganaran más de 5 salarios mínimos y $20 \%$ a los que perciban por encima de 10 
salarios mínimos. Aun así, las autoridades afirmaban que las medidas tomadas no eran suficientes para amortiguar el daño económico y al mismo tiempo advertían con mayor insistencia sobre la necesidad de cambios significativos en el aparato estatal. Más que nunca, el debate de la reforma se instalaba en el escenario político y se hacía eco en la opinión pública.

Como ya se mencionó anteriormente, la idea central del discurso reformador proveniente del gobierno, gira en torno a la urgencia de 'achicar' el Estado como manera de ordenar mejor el gasto público, gasto que, según los ideólogos de dicha propuesta se encuentra despilfarrando dinero en el pago a un número desproporcional de funcionarios estatales, entre ellos una considerable parte serian supuestos 'planilleros', circunstancia que exige al Estado gastar el $76 \%$ de la recaudación tributaria en financiar salarios de la administración pública (Presupuesto General de la Nación, 2020) y obligando de esta forma a que la cobertura en algunos campos sociales sean limitadas por la falta de recursos.

Si bien en las instituciones públicas han existido casos de prebendarismo y cobros excesivos en materia de sueldos, lo cierto es que, proponer una reforma bajo el solo efecto de descongestionar el Estado, suena más bien a un fuerte impulso neoliberal antes que, a un proyecto de contención de la pandemia, independientemente de las intenciones que se tengan en redireccionar dichos gastos en salud y educación, las soluciones que presentan no dejan de ser superficiales y mucho menos se aproximan a ser consideradas estructurales. Por supuesto que se debe poner fin a los viejos vicios de la corrupción y el clientelismo en el sector público, pero existen otras alternativas para fortalecer la inversión social y no es precisamente solo reduciendo el Estado, sino implementando nuevas medidas tributarias.

\section{De la justicia fiscal a una justicia social}

Se entiende la necesidad de pagar los 1600 millones de dólares de la deuda contraída, pero hasta el momento, ninguna autoridad estatal del oficialismo, se ha animado a comentar que podríamos cubrir la cuarta parte del préstamo emitido, si aumentáramos el impuesto a la exportación de la soja a un $15 \%$ y recaudar con ello unos 400 millones de dólaresanualmente (Sarate, 2018). De la misma manera podríamos apuntar a otros campos comerciales como por ejemplo a los productos azucarados, las comidas chatarras, el alcohol o el tabaco (Serafini, 2020). Sobre este último rubro, la recaudación en impuestos por el consumo de cigarrillos en Paraguay ronda los aproximadamente los 318 millones guaraníes anuales, un valor que llega a cubrir sólo al $20 \%$ de los gastos médicos directos causados por el tabaco al sistema nacional de salud (Bardach et al., 2018).

$\mathrm{Si}$ aumentáramos el impuesto del tabaco en un $50 \%$ al precio de los cigarrillos, lo cual es una estrategia ya implementada en otros países, se lograría evitar una considerable cantidad de muertes y enfermedad, además de recaudar el equivalente a las pérdidas generadas por el tabaquismo anualmente. Pero en el caso que no fuese tan simple la cuestión y se diera un aumento solo del $20 \%$, de igual forma se estaría percibiendo aproximadamente 656 millones de guaraníes por año, un monto no menor si se tiene en cuenta la necesidad del contexto sanitario (Bardach et al., 2018).

Como podemos apreciar, existen varias opciones que nos permitirían recaudar e invertir posteriormente, pero aquí radica el problema, la presión tributaria en el Paraguay es sumamente baja y desigual entre los diferentes sectores en comparación a los demás países de la región. A modo de ejemplo, mientras que el promedio de América Latina en el 2014 era del 21,7\% (OCDE, 2016, p. 41), en Paraguay en el 2015 fue del 12,5\% (SET, 2016, p. 1). Se estima que factores como las bajas tasas de impuestos, el alto nivel de evasión, los 
gastos tributarios elevados, entre otras cosas, debilitan la presión tributaria del país (Borda \& Caballero, 2016) y esto a la vez impide que el Estado ofrezca de manera adecuada los bienes públicos por la falta de recursos.

El inconveniente con los reformistas neoliberales es que ellos apuntan $y$ discuten temas que nada tienen que ver con los problemas reales de fondo. Colocan al funcionario público como el principal culpable del limitado alcance de la cobertura estatal, cuando en realidad la poca inversión social también está relacionada directamente a la débil carga impositiva en algunos rubros, rubros que podrían aportar mucho más al Estado en materia de tributación y con ello aumentar la inversión social. Evidencia científica hay, porque existen un montón de estudios sociológicos y económicos que demuestran la viabilidad de implementar nuevas medidas tributarias, lamentablemente al momento de tomar decisiones no son tenidas en cuenta o simplemente son ignoradas porque atentan los intereses de sectores que han captado el poder político.

Es legítima la intención de ahorrar recursos, principalmente si están siendo derrochados en sueldos de funcionarios privilegiados, pero es necesario preguntarse también ¿cuáles son los espacios prioritarios donde faltan agentes y donde hay demás? La cosa no es tan simple como lo plantean algunas figuras políticas. Un desmantelamiento acelerado e indiscriminado de la estructura estatal puede implicar una mayor desprotección de la ciudadanía y la expulsión de personal, especialmente si se trata del personal técnico capacitado, puede limitar o anular la capacidad estatal de formular e implementar políticas estratégicas.

Es cierto que urge un recorte de los salarios desproporcionales que existen en las instituciones públicas, en particular en las binacionales, también es cierto que se necesita poner fin a los cargos prebendarios, pero, no se puede considerar que exclusivamente con estas reducciones se podrá subsanar todos los efectos económicos de la crisis sanitaria. Es menester además de reducir los exorbitantes salarios, encontrar otras vías de obtención de ingreso fiscal, y para ello es necesario pensar en un nuevo paquete tributario, en particular revisar la situación impositiva actual del agronegocio, del tabaco y del alcohol.

Una reforma con rostro humano, exigirá gastar más y mejor el dinero público en programas de salud, educación y protección social. De igual modo, en el área de la infraestructura, será urgente invertir más en caminos rurales, viviendas, agua y saneamiento. En este sentido, los recursos adicionales obtenidos de un aumento en la presión tributaria brindarán al Estado paraguayo la capacidad para encarar estos desafíos y muchos otros.

Además de rever las políticas tributarias, la reforma tendrá que lidiar con un posible aumento de la pobreza. Son muchas las empresas, medianas y pequeñas que han quebrado o han visto disminuir sus ingresos a causa del distanciamiento social, teniendo efecto directo en miles de personas que quedaron sin empleo, esto hace que la pobreza existente se profundice y la calidad de vida de quienes ya antes la tenían difícil, hoy en día deba empeorar. En ese contexto, se estima que la pobreza en la región aumentaría un 4,4\% durante el 2020 al pasar de una tasa del 30,3\% al $34,7 \%$, lo que representa un incremento de 29 millones de personas en situación de pobreza (CEPAL, 2020).

Los números en el país mucho antes de la crisis sanitaria no eran los más favorables. En Paraguay más de $23 \%$ de la población es pobre, lo que equivale a más de un millón seiscientas mil personas que viven en hogares cuyos ingresos son inferiores al costo de una canasta básica de consumo, según la última encuesta permanente de hogares del 2019. Los datos de la Dirección General de Estadísticas, Encuestas y Censos (DGEEC), hasta el último trimestre del 2019, advirtió que la tasa de desempleo en el país rondó 
alrededor del $5,7 \%$ equivalente a 209.538 personas, es el mayor número registrado en los últimos 2 años, lo que demuestra la poca efectividad de las políticas públicas orientadas a crear puestos de trabajo. A este panorama que ya venía siendo preocupante, se le suma el impacto de la crisis actual, pues, tan solo hasta la segunda quincena de abril, unas 1780 empresas habían solicitado al Ministerio del Trabajo el cese temporal o definitivo de contratos por la pandemia del COVID-19, lo que implica que unas 60.000 personas estarían quedando desocupadas, en ese sentido, el instituto de previsión social (IPS) informó que ya ha sumado unos 30.000 pagos por subsidios a trabajadores que dejaron sus puestos.

Si de la experiencia aprendiésemos, la futura reforma debería contemplar, por un parte, el seguro de desempleo, esto ayudaría quienes pierden sus trabajos por una eventual crisis similar a la actual, no sufrir consecuencias bruscas al ser interrumpido su actividad laboral, es decir, que su calidad de vida no se vea afectada considerablemente al momento de encontrarse desocupado. Por otra parte, es necesario fortalecer el mercado local, el consumo interno, asegurando la soberanía alimentaria, de la mano de una verdadera y justa reforma agraria que ponga fin al conflicto de la tierra y al monopolio de la propiedad privada sobre la misma. Aquí, una reforma tributaria sería oportuna para mejorar el acceso a viviendas sociales y equipamientos tecnológicos para la agricultura familiar. Las inversiones en estos campos son imprescindibles para disminuir la pobreza, la desigualdad y mejorar la calidad de vida de la gente.

Otro punto a poner énfasis en la futura reforma, es en la situación de la informalidad, la economía no formalizada priva a miles de personas de contar con asistencia médica, equipos de protección adecuados, salario digno, jubilación, entre otras cosas. Según datos de la Asociación Pro Desarrollo Paraguay, la cobertura social en el país es apenas del $20 \%$ y en cuanto a la economía subterránea, representa aproximadamente el $40 \%$ del producto interno bruto, mayormente son cuentapropistas no profesionales y empleados de microempresarios, ocupados en actividades como el comercio y servicios (Masi, 2002). Las consecuencias de la informalidad se pueden apreciar en los altos niveles de subcontratación. Esta subcontratación por lo general se da sin obligaciones de coberturas legales como seguridadsocial,vacaciones, bonificaciones, jubilación o el salario por debajo del mínimo establecido. Las consecuencias de este fenómeno, además de afectar la productividad del país, también tienen connotaciones profundamente sociales pues la alta informalidad está relacionada con otros atributos desfavorables como alta desigualdad, corrupción e instituciones débiles.

El incumplimiento de las leyes laborales, suele ser uno de los puntos más visibles de la informalidad, pero las consecuencias más duras se dan cuando afectan la calidad de vida las personas (Olmedo Colarte, 2019). Por esta razón, el análisis de la informalidad en la próxima reforma, no debe centrarse solo en el aspecto jurídico, pues claramente se tratan de problemas que invaden el campo social.

\section{Conclusión}

Por supuesto que falta reformar el Estado, pero se necesita cambios que superen la perspectiva neoliberal y ponga atención a la universalidad de los derechos humanos básicos, principalmente a los que se refieren a salud, educación, alimentación, reforma agraria, trabajo, seguridad social y por sobre todo es urgente una reforma tributaria con justicia fiscal, en donde las ganancias exorbitantes paguen el impuesto correspondiente a sus ingresos y a los daños que causan.

La reforma debe contemplar un programa para el desarrollo integral de las personas, con perspectiva en derechos humanos, justicia social e inclusión, particularmente de las comunidades campesinas, de los pueblos originarios, 
los asentamientos suburbanos y las personas con discapacidad. En esa tarea, la sociología y otras disciplinas juegan un rol fundamental como guía, así como la ciencia médica cargó sobre sus hombros gran parte de la contingencia de la pandemia, las ciencias sociales deben asumir la responsabilidad de pensar nuevos y mejores escenarios a futuro.

Está por demás decir, que el debate no se agota aquí, al contrario, de ahora en más se abre un abanico de discusiones sociológicas, económicas y políticas en torno al tema. Otros estudios han abordado y abordarán con mayor detalle cada punto tratado en este ensayo, no obstante, en lo que toca a esta breve y franca reflexión sociológica, los números y los posibles escenarios no mienten, existe un panorama totalmente a favor de nuevos programas tributarios que ayudarían contener los efectos provocados por la pandemia del COVID-19.

\section{Referencias}

Bardach, A., Cañete, F., Sequera V. G., Palacios, A, Alcaraz A., Rodríguez, B., Caporale, J., Augustovski, F., \& PichonRiviere, A. (2018). Carga de enfermedad atribuible al uso del tabaco en Paraguay y potencial impacto sanitario y económico del aumento del precio a través de impuestos. Rev Peru Med Exp Salud Pública, 5(4), 599-6o9. 10.17843/ rpmesp.2018.354.3708

Borda, D., \& Caballero, M. (2016). Eficiencia y equidad tributaria: Una tarea en construcción. Paraguay Debate/Centro de Análisis y Difusión de la Economía Paraguaya, CADEP.

CEPAL. (21 de abril de 2020). Pandemia del COVID-19 llevará a la mayor contracción de la actividad económica en la historia de la región: caerá $-5,3 \%$ en 2020. https://www.cepal.org/es/ comunicados/pandemia-covid-19llevara-la-mayor-contraccion-laactividad-economica-la-historia-la

Dirección General de Estadísticas, Encuestas y Censos, DGEEC. (2019).
Encuesta de Hogares 2019. http:// www.stp.gov.py/vi/dgeec-publicolos-principales-indicadores-depobrezamonetaria-del-ano-2019/

Ley de Presupuesto General de la Nación 2020. https://www.bacn.gov.py/leyesparaguayas/9115/ley-n-6469-apruebael-presupuesto-general-de-la-nacionpara-el-ejercicio-fiscal-2020

Masi, F. (2002). Sector Informal y cuentapropismo en el Paraguay. Economía y Sociedad: revista de análisis, (7).

Olmedo Colarte, C. A. (2019). La dimensión socio-política de la informalidad económica en Paraguay. Explorando la cultura del incumplimiento.

Organización para la Cooperación y el Desarrollo Económicos, OCDE. (2016). Revenue Statistics in Latin America and the Caribbean 2016. OECD Publishing.

PRO Desarrollo Paraguay. (2019). La eficiencia del gasto público y su relación con la informalidad. https://www.pro. org.py/contenido/2019.pdf

Sarate, J. (2018). Abdo Benítez necesitará del impuesto a la soja para gobernar. En Con la Soja al cuello. Asunción: BASE IS.

Serafíni, V. (2020). Pandemia y salud en Paraguay: Dos mitos sobre el papel del estado y el desarrollo. Centro de Análisis y Difusión de la Economía Paraguaya.

Subsecretaría de Estado de Tributación, SET. (2016). Presión tributaria. http:// www.set.gov.py/ 
Ovando, L. A. El papel de las Ciencias Sociales frente a la intención de una reforma neoliberal del Estado paraguayo.

\section{Sobre el Autor}

Luis Alexander Ovando

Estudiante de la Facultad de Ciencias Sociales de la Universidad Nacional de Asunción. 